\title{
ICT IN INSTRUMENTATION AND MEASUREMENT: EDUCATION AND SCIENTIFIC PUBLICATIONS
}

\author{
Remigiusz J. Rak \\ Warsaw University of Technology, pl. Politechniki 1, 00-661, Warsaw, Poland, rrak@okno.pw.edu.pl
}

\begin{abstract}
The article gives a review of reasons for developing and adopting the new ICT tools in teaching and scientific research in the field of Instrumentation \& Measurement. It includes a description of the Internet-and multimedia-based educational model (e-learning). Then follows a description of the structure and tools of the modern scientific electronic book (e-book). The last part is oriented on Virtual Instruments, remote experiments and Virtual Laboratory.
\end{abstract}

Keywords: ICT, e-learning, e-book, virtual instrument, distributed systems, virtual laboratory, distance learning.

\section{INTRODUCTION}

Most of the recent trends in the area of Instrumentation \& Measurement come from quite different branches of science and technology. The most important between them is Information and Communication Technology (ICT). ICT joins together: information, computers, computer science, computer engineering and communication. ICT is growing very expansively. It creates new, great possibilities and offers the new tools. At the same time ICT creates new difficult tasks and challenges. But on the other hand expansion of a new technology very often exceeds ideas, expectations and imaginations of a number of people. People observe a growth of science and technological progress with a fear and see there a source of threat. Some people are forced to change their jobs many times in their life. More and more people who cannot work all the life with a high activity fall behind. Education is a very serious remedy for those fears. So that, generality and necessity of education should have the highest priority level in the era of Information Society.

New developments in the area of ICT have enriched a traditional classroom with the new tools, improving learning quality in both residential universities and geographically dispersed learning individuals (distance learning). Of all the technical innovations, computer and the Internet have become the indispensable tools in introduction of the new technology to education, and its growing impact on the future of the educational model is inevitable. In the behaviorism model a computer and Internet were considered as a "blackboard" with higher functionality. In the constructive model they both, play a role o the meaningful cognitive tools. All the ICT tools can be effectively introduced into the Instrumentation and Measurement teaching in a very easy way.

Let us start from the lowest level. The contents of the particular courses, as well as scientific books, can be prepared in the electronic version (e-books) and stored on CD-ROM. Certainly, the same content can be placed on the web sites, available via Internet. The electronic books have the advantage of presenting the whole material of a single subject on one $\mathrm{CD}$. Because of nearly unlimited capacity, besides traditional educational content it can include: auxiliary software, selected set of scientific publications and „source readings”, addresses and links to other knowledge sources (e-libraries, archives), animations, simulations of experiments and tests.

The critical element of testing theory throughout experiments cannot be missing in this model of teaching. The missing link is the possibility to carry out physical experiments over the web, fully integrated with other media for delivering classroom content and scientific e-books, worldwide. By supplementing classroom teaching with web-based remote experiments, the student should be able to interact with physical systems, much in the same way as modern experiments are carried out today, under computer control. Virtual Laboratories accessible from the Internet provide meaningful enrichment to the educational experience that is hard to obtain from other video based remote teaching methodologies. 


\section{ELECTRONIC BOOK}

As it has been mentioned above, the didactic materials of the particular courses in the I\&M area can be prepared by professors and experienced lecturers in the form of electronic lectures (books) and stored on CD-ROM. The electronic books have the advantage of presenting the whole material of a single subject on one $\mathrm{CD}$. The cost of multiplying it is relatively low. Creation of the material can be done with the Dynamic HTML technology (HTML, Cascading Style Sheets, Java Script and FrontPage tools). Because of nearly unlimited space, besides traditional educational content it can include [2][3]:

- Auxiliary software,

- Set of publications and ,source readings”,

- Addresses and links to other knowledge sources: e-libraries, archives, collections,

- Questions/answers and tests,

- Animations,

- Simulations of experiments.

The material of an electronic book should be divided into three main parts:

- Introductory part,

- Learning Units,

- Exam requirements.

The introductory part includes:

- Authors' note, which describes course objectives.

- Requirements for computer - includes description of requirements for computer.

- How to use an electronic book - step-by-step instruction of how to use the material stored on the CD-ROM.

- What to know, to understand? - conditions for understanding the didactic materials: minimal level of knowledge.

The Learning Unit should include a series of basic didactic capters that should be learnt in a suggested order. Every learning unit includes several basic elements like:

- Introduction, which presents the aim of the particular Unit.

- Knowledge segments, which include basic didactic material.

- Problems, this part includes examples of partly solved problems and tasks to be finished by the student himself.

- Glossary includes new terms and definitions.

- Bibliography includes a list of important publications for further reading.

The Exam requirements unit should be included only if the given course ends with an examination.

A proposed structure of the electronic book is presented in fig.1.

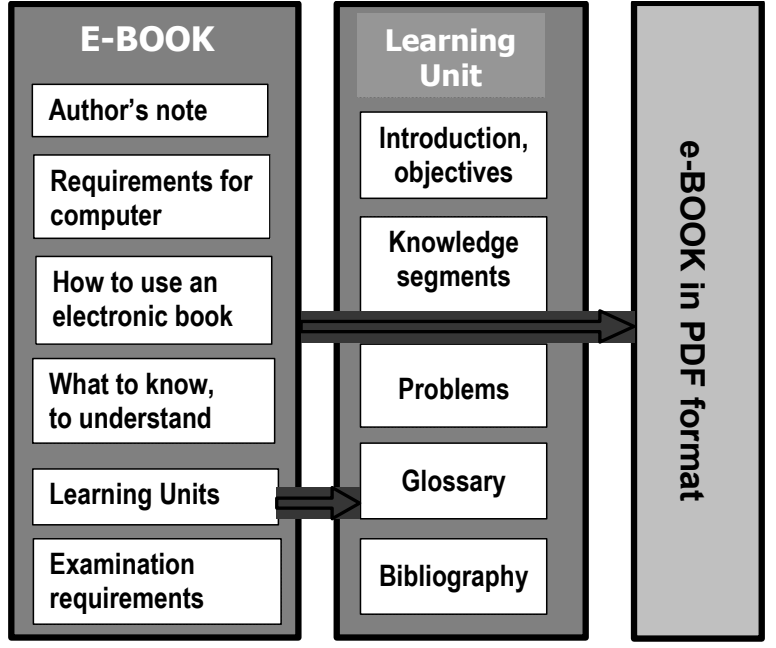

Fig. 1 - A proposed structure of electronic book

The tools which can be implemented in the creation of an e-book, are divided into three main categories:

Traditional tools:

- Texts,

- Fonts: bold, italic, color etc.,

- Equations,

- Drawings,

- Photos,

- Background color, texture.

Multimedia tools:

- Text comments,

- Audio comments,

- Video comments,

- Animation of drawings,

- Animation of presentations.

Advanced tools:

- Generators of tests,

- Local simulations (tool software on the own PC),

- Distance simulations (the tool software on the application server at the university),

- Simulated experiments,

- Remote experiments.

Java and Flash are very meaningful software tools in the area of animations and simulations. Java programming language is a very useful tool in writing multimedia network applications. Java program can be run under any operation system. Java applets can be introduced into an HTML text of any e-book. Java is able to enriched multimedia content of web sites throughout the animations, advanced graphic, sound and images with no any need for introducing additional applications available throughout the WWW viewer. A very complex program can be distributed throughout the polymorph network, Internet with no necessity of knowing what kind of operating system is used by the student. 
An example of Java applet is presented in fig. 2. It represents a simple digital scope attached to some virtual sinusoidal signal source.

An example of Java applet, presented in fig.3, is prepared as a set of instruments (generator on the top and scope on the bottom) connected together and creating a measurement system for investigating of an amplifier frequency response. The frequency response can be investigated manually - what is certainly intentional.

The second, not less helpful tool in the area of animations destined to web sites, is Flash from Macromedia. It generates small capacity files, acceptable by the all web sites viewers. The Flash format, with ".swf" extension, is based on the vector graphic, where instead of keeping information about each image pixels, like in bitmap, there are used mathematical formulas describing shape, color and layout of the all object. Thanks to that, the created file has smaller capacity and even complex animations are lauded to the viewer very fast. The implementation of FLASH animation is a very simple process, available to everyone. An example can be observed in fig.4. On the monitor screen we can see two instruments: function generator (on the bottom) and scope (on the top). They are connected together. The output signal from generator can be seen on the scope display.

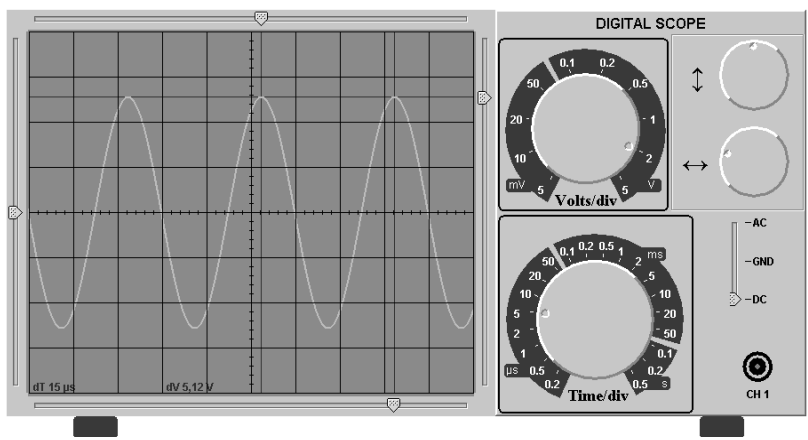

Fig. 2 - A digital scope in the form of Java applet

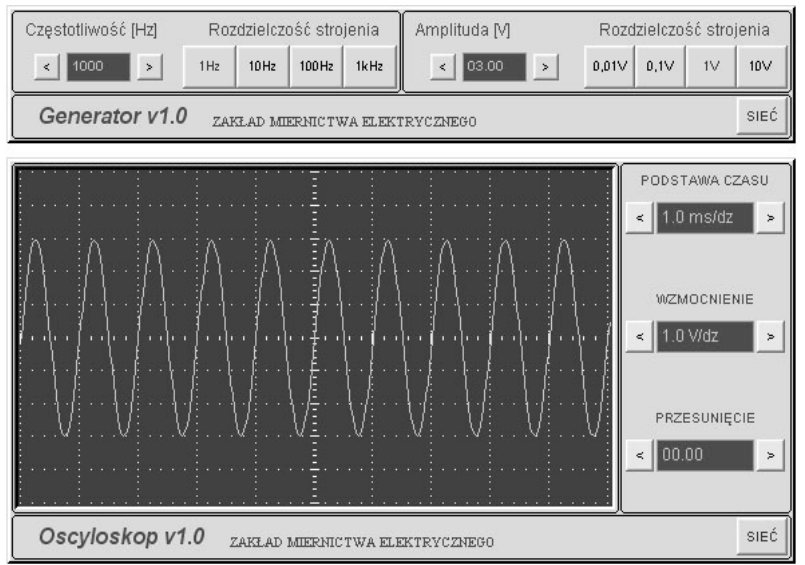

Fig. 3 - A set of instruments in the form of Java applet

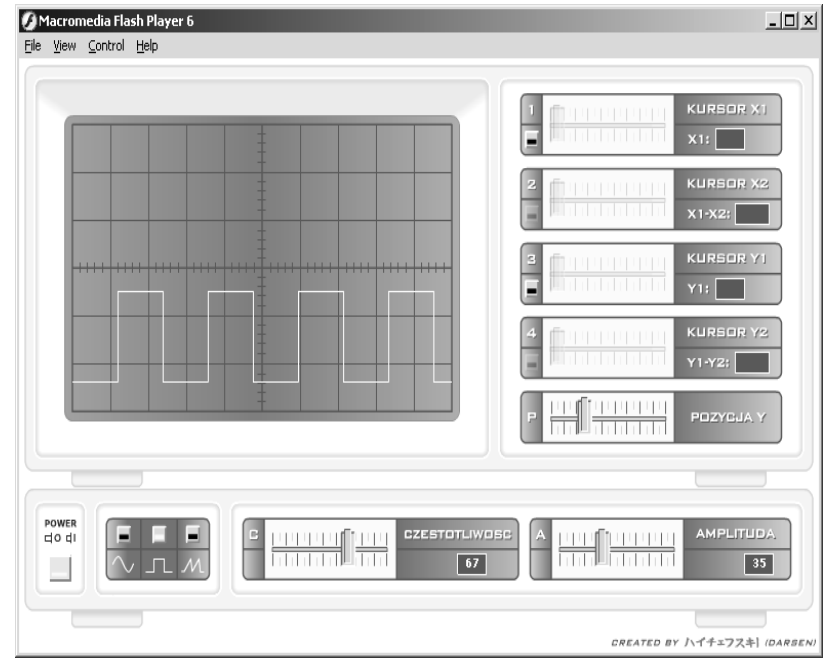

Fig. 4 - A set of instruments as a Flash module

All the simulations of digital instruments described above are strongly connected to the idea of Virtual Instrument.

\section{VIRTUAL INSTRUMENTS}

The clearest definition of Virtual Instrument has been prepared by National Instruments:

"A layer of software and/or hardware added to a general-purpose computer in such a fashion that users can interact with the computer as thought it were their own custom-designed traditional electronic instrument."

Very interesting idea has been exposed by $\mathrm{H}$. Goldberg [5]:

What is virtual instrument? - A virtual instrument is composed of some specialized subunits, some general-purpose computer, some software, and a little know how. Where is it? - Its various parts can be separated all over the workspace or, using the Internet, anywhere in the world. With the introduction of some of the newest remote radio communication protocols such as "Bluetooth", the components needn't even be physically connected to each other.

The main categories of Virtual Instruments are presented below [1][9][10]:

- Computer controlling GPIB or RS232 instruments, with a graphical front panel on the computer screen to control the instrument;

- Plug-in DAQ board or a PXI module instead of an external instrument, with a graphical front panel on the computer screen to control the instrument;

- Graphical front panel with no any physical instrument connected to the computer. Instead, the computer acquires and analyzes data from files or from other computers on a network, or it may even calculate its data mathematically to simulate a physical process or event. 
The most popular way of programming Virtual Instruments is based on the high-level tool software. With easy-to-use integrated development tools, design engineers can quickly create, configure and display front panels of virtual instruments as a kind of graphical user interface enabling to conduct measurements in user-friendly form. The most known, popular software tools are as follows:

- LabVIEW (National Instruments) - is a highly productive graphical programming language for building data acquisition and instrumentation systems (Virtual Instruments). To specify our system functionality we intuitively assemble block diagrams - a natural design notation for engineers. Its tight integration with measurement hardware facilities rapids development of data acquisition, analysis and presentation solutions.

- LabWindows/CVI (National Instruments) - is a Windows based, interactive ANSI C programming environment designed for building virtual instrumentation applications: $\mathbf{C}$ for Virtual Instruments. It delivers a drag-anddrop editor for building user interfaces and a collection of automated code generation tools as well as utilities for building automated test systems, monitoring applications or laboratory experiments.

- HP VEE (Hewlett-Packard) - is a kind of Visual Engineering Environment, an iconic programming language for solving engineering problems. It also gives the ability to gather, analyze and display data without conventional (text-based) programming.

- TestPoint (Keithley) - is a Windows based, object-oriented software package that contains extensive GPIB instrument and DAQ board support. It contains a novel, state-of-the art user interface that is easy to use. Objects, called "stocks" are selected and dragged with a mouse to a work area (panel). Logic flow is easily established with a point and drag action list.

The power of tool software lies in libraries. Library functions enable:

- An access to instrument Interfaces (GPIB, RS232/485, PXI, DAQ)

- Full control of autonomic Instruments (Instrument Library),

- Creation of Graphical User Interface GUI (User Interface Library, Formatting and I/O Library),

- Digital Signal Processing DSP (Advanced Analysis Library),
- An access to global computer network INTERNET (TCP/IP, DataSocket, ActiveX),

- Inter-process data exchange DDE (Dynamic Data Exchange).

The ideal tool enabling easy control of programmable instruments is specialized command set, high level language, called SCPI (Standard Commands for Programmable Instruments). SCPI dramatically decreases development time and increases a readability of test programs. It has its own set of required common commands in addition to the mandatory IEEE 488.2 common commands and queries. Although IEEE 488.2 is used as its basis, SCPI defines programming commands that we can use with any type of hardware or interface links. It has quite opened structure. The SCPI Consortium continues to add commands and functionality to the SCPI standard. For example, the following command programs a digital voltmeter (or even a digital scope) to configure itself to make a DC voltage measurement on a signal of $30 \mathrm{~V}$, with a 0.002 resolution:

\section{:MEASure:VOLTage:DC? 30, 0.002}

The most modern potential feature of Virtual Instrument is its possibility to estimate the current measurement uncertainty in on-line mode. Such a procedure can be attached to the main algorithm for MEASURAND recovery.

The most popular category middle) includes a DAQ board for data acquisition. The block diagram of such an instrument controlling data coming from the real measuring object is presented in fig.5.

In a case of introducing a network board into the PC our VI can be exposed to the LAN or even to the Internet.

Another sophisticated feature of a Virtual Instrument is exposed in fig.6. As it can be seen it's main functions (data acquisition, data analysis and data presentation) are physically distributed between three independent computers.

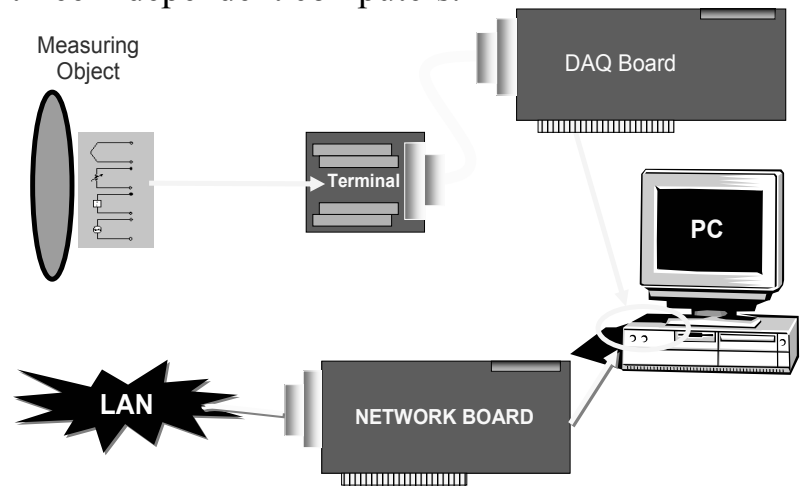

Fig. 5 - Block diagram of the VI based on the DAQ board 
An example of reconstruction of the real instrument (function generator Agilent 33120A) prepared under LW/CVI is presented in fig.7.

As a matter of fact it is a kind of simulation module prepared in LabWindows/CVI environment. This virtual front panel can be certainly used to control a real Agilent 33120A instrument attached to the controller (PC equipped with GPIB board).

Another several more examples of VI front panels are presented in the following figures (8-10).

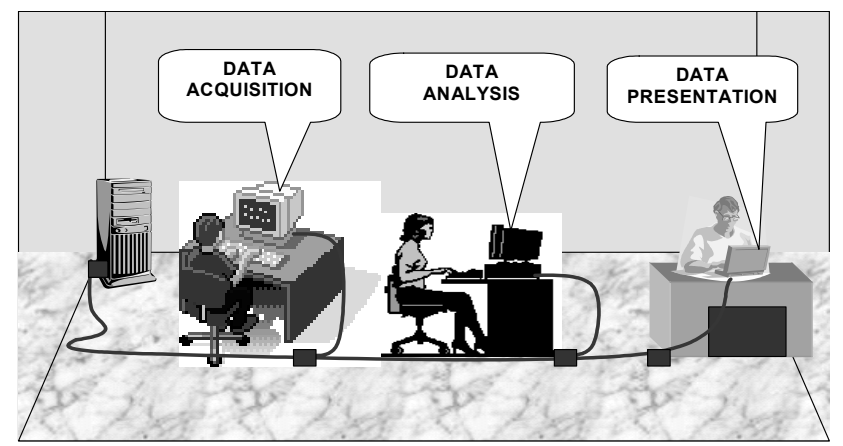

Fig. 6 - The idea of distributed instrument

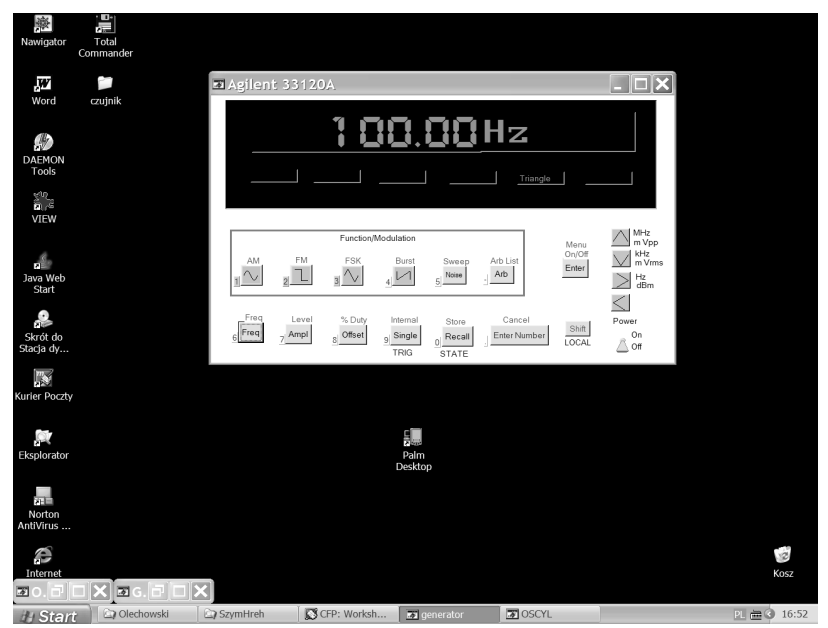

Fig. 7 - Reconstruction of the real instrument function generator Agilent 33120A

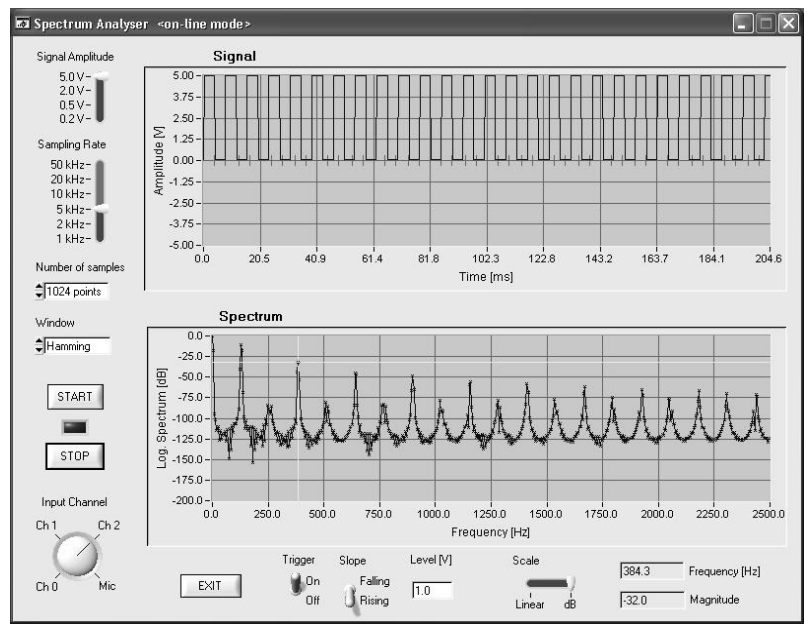

Fig. 8 - Spectrum Analyzer

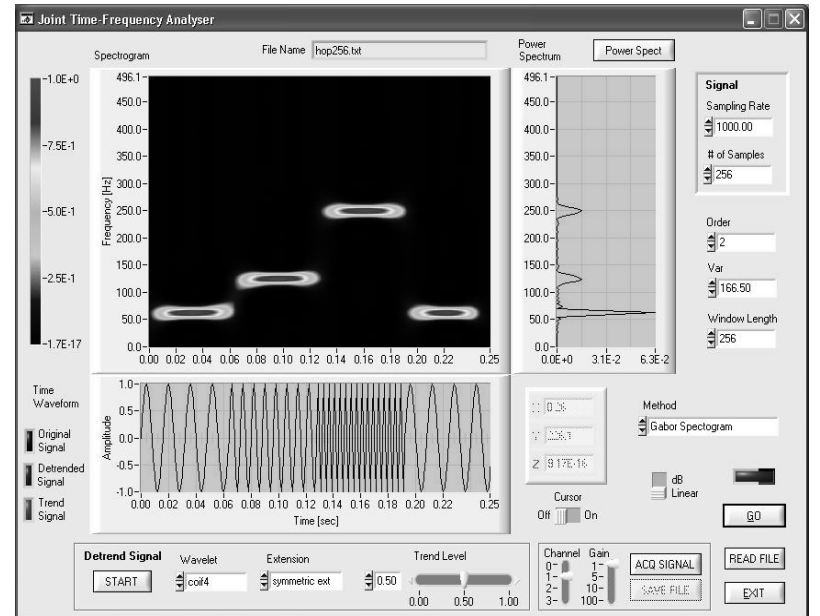

Fig. 9 - Joint time-frequency analyzer

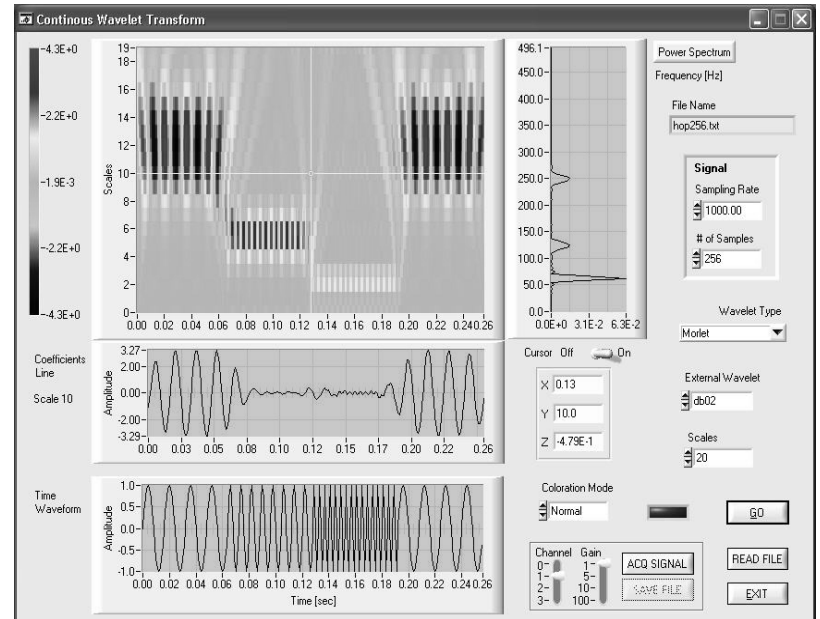

Fig. 10 -Wavelet based Time-Scale Analyzer

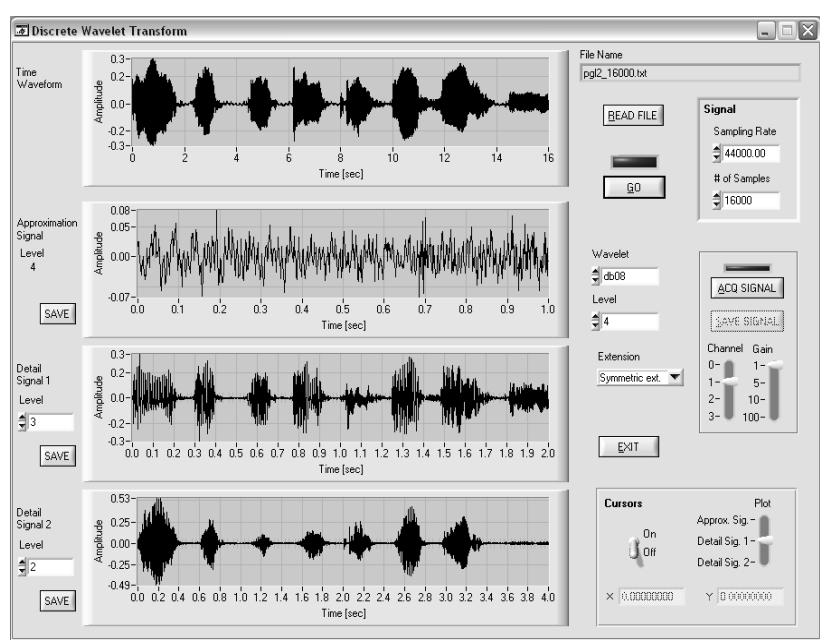

Fig. 10 - Discrete Wavelet based Signal Analyzer

Virtual Instruments replaced expensive and complicated Intelligent Instruments the main part of didactic laboratories. This has simplified the process of designing, conducting and modernizing these laboratories. The Graphical User Interface, which resembles real instrument, enables the users of conventional instruments to understand and operate new instruments intuitively. 


\section{VIRTUAL LABORATORY}

As it has been already mentioned above, in scientific and engineering fields, the crucial phase of the learning process is the practical training activity helping to assure a good knowledge transfer from teacher to students. Teaching in the area of Instrumentation \& Measurement engineering, the students have to work on real instrumentation in conditions as realistic as possible. They should also have the opportunity to repeat the same experience several times and even learn in a trial and error mode.

As it has been already shown (fig.5) VI can be exposed to the LAN or even to the Internet in a quite easy way. It is possible to give a user a free access to the measurement results as well as let him to control the instrument in remote manner [8].

The main drawbacks of introducing those elements into the laboratory experiments are following [1][11]:

- high cost of measurement instrumentation,

- high cost of management of experimental laboratories,

- reduced number of laboratory technical personnel and their expensive updating,

- increasing number of students.

There is only one practical solution of this problem: To build a Virtual Laboratories (VL) and to share the limited resources of one or more laboratories among a large number of teachers and students over Internet.

Remote control of experiments and equipment over the web is an idea that is just being explored. Different tools are now becoming available for remote control of instrumentation using network communication. Several demonstrations of camera control and data acquisition as well as simple experiments have already been made. Our knowledge concerning processes resulting from experiments, ability to control these processes and a set of tools needed for digital recording and transmission are good enough to introduce a new model of laboratory - Virtual Laboratory. The most important elements of Virtual Laboratory are: Virtual Instruments and Distributed Measurement Systems.

These features of Virtual Laboratory involve:

- use of mass storage to archive/back up data, which is delivered from different hosts,

- sharing of software resources that are installed in a network,

- functional integration of distributed measurement resources,

- open access,

- concurrency and calibration,

- fault tolerance.
The ICT tools which are very helpful in a case of Virtual Laboratory design are listed below:

- Internet Protocols: IP, UDP, TCP;

- SOCKETS) - available under Unix (and Windows 9X/NT as winsock) method of network communication between applications;

- „Client-Server” architecture: Server must open the socket and wait for connection. To the open socket is attached a certain number called port. Client have to know network address of the computer (server) as well as the port number;

- JAVA : Object oriented programming language which enables creation of the system software throughout the set of cooperating independent components (objects);

- Objects: real or virtual instruments, device drivers, servers, clients, and at last abstract objects like mathematical, logical functions.

The idea of VL is presented in fig. 11.

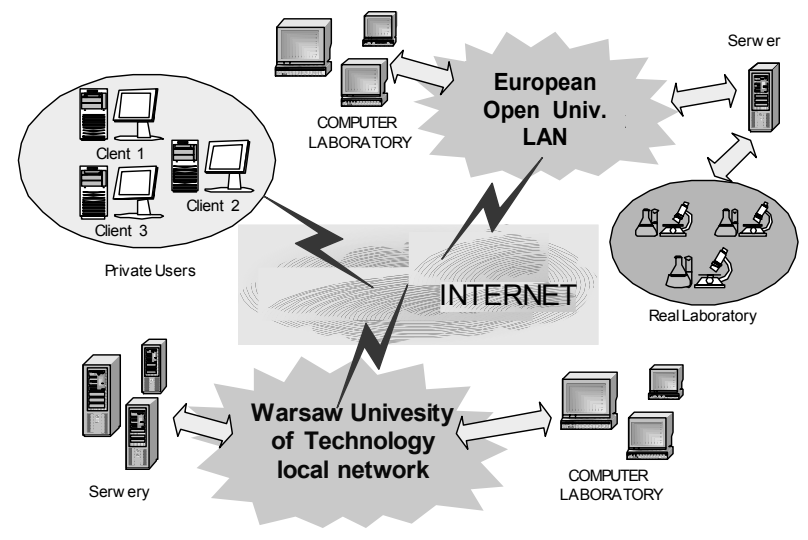

Fig. 11 - The idea of Virtual Laboratory

The proposed architecture of virtual laboratory (fig.12) that implements both local area network and the Internet is characterized by features that are, in general, assigned to territorially distributed systems [6]. To the key elements of the proposed system architecture belong [11]: the main server, the so called system manager and measurement servers.

Managing server is equipped with WWW server and database server, compatible to SQL. Measurement server can be equipped with GPIB interface, DAQ card or PXI modules. Server software should enable the user to conduct ondemand measurements and measurements that take place in the so called 'gentle' time. The on-demand measurement is based on question-answer principle. The user defines parameters of the measurement and then sends a 'demand' request to execute the measurement. While measurements are conducted the user interacts with virtual instrument, as if he was interacting with the real instrument. During measurements the user can freely change parameters and functions of the instrument and observe results. 


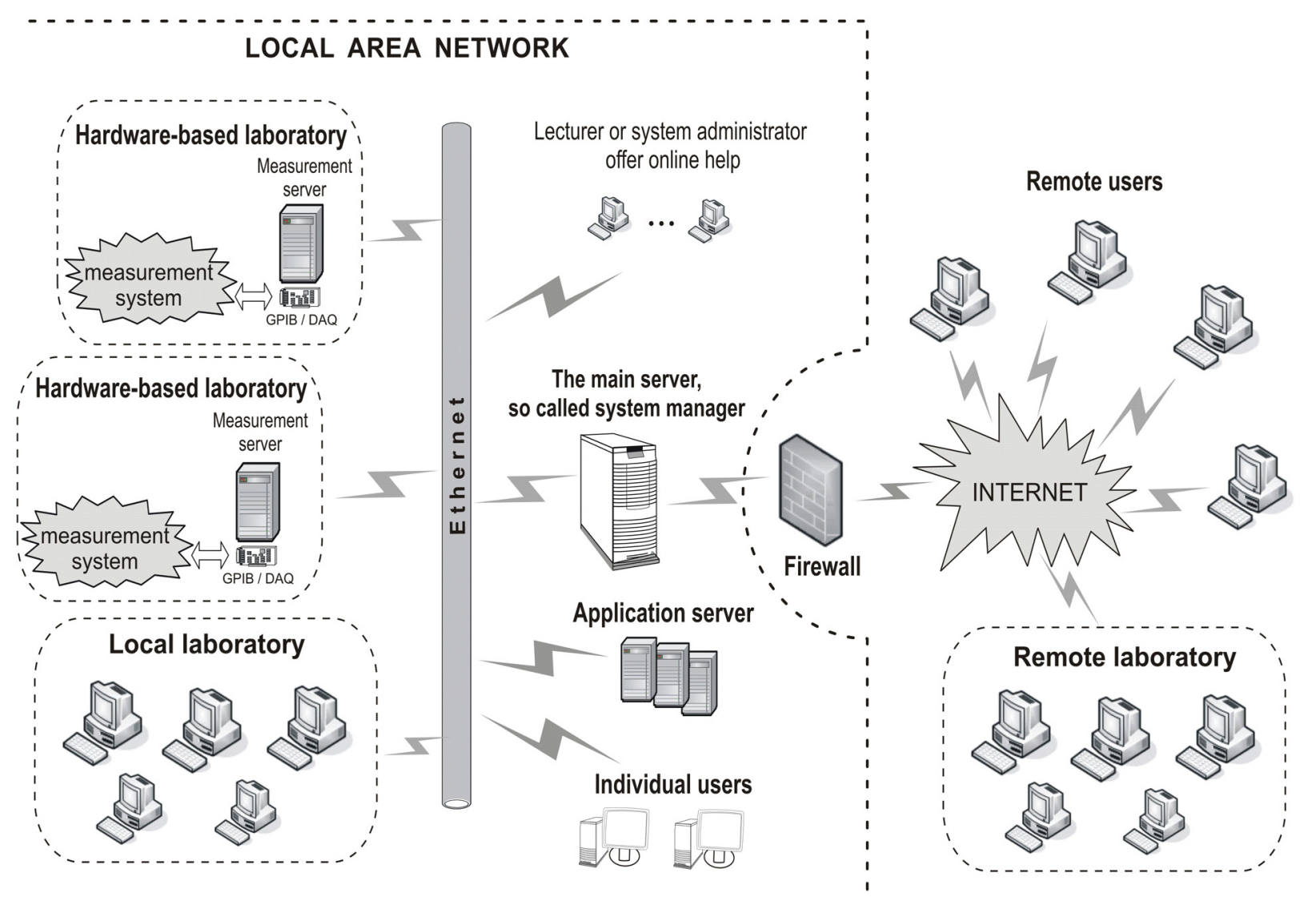

Fig. 12 - A typical structure of Virtual Laboratory

It is important that students can understand the essence of events that take place in a measurement object, and familiarize with measurement systems and techniques. This should be done without limiting the number of accessible instruments of a given measurement unit. Moreover, it is vital that students can freely configure a measurement system, and even build a faulty circuit. This should enable a student to better understand the problem on the basis of 'trial and error' method. Although virtual laboratory software should block any operations which may destroy some hardware parts of the measurement system. More experienced and skilled students will be given more freedom in conducting their experiments.

Another important function of the virtual laboratory is an access to the experiment in the 'offline' mode. This could allow the user to learn, understand and partially solve problems that he might encounter in practice. Such a system should enable to design and then store the measurement unit architecture. This would minimize the time needed for remote access to real instruments that are essential to conduct experiments. Yet another useful function of a virtual laboratory is a possibility of simulating simple experiments that can be used to present a problem discussed during a lecture. This function may be very beneficial for users that are just about to learn how to use a virtual laboratory.

\section{CONCLUSION}

Introduction of e-books into the Instrumentation and Measurement teaching process and placing the measurement instrument in a distributed system based on the Internet enables creating advanced and flexible systems that make possible to support learning processes.

An important objective for the future is a remote Virtual Laboratory, a very useful tool for teaching purposes in distance learning. Students can access Virtual Instruments via a geographic network and directly carry out real experiments by using of a simple standard commercial Internet Web browser. In this way, a more complete educational proposal, can be offered by several laboratories specialized in different measuring fields. The remote laboratory concept allows measuring resources located at different geographically remote sites to be utilized by a wide distribution of students.

Experimental studies of physical events or objects with the use of measurements instruments help students to understand measurement procedures and measurement system structures. Thus, virtual 
laboratory should be treated as a very attractive tool supporting education. Remote access to laboratories assures rational managing of expensive and unique measurement equipment. In other words, it facilitates integration of measurement resources.

It is predicted that in the future the virtual reality techniques will develop so far that the Internet browser will be replaced by 3D interface. The user will have an impression that he is walking inside a laboratory where all the instruments and units will look like real ones. The user may be even tempted to touch these instruments.

Another objective for the future is the establishment of a network of universities offering distance education. This would lead to creation of a new model of distance education based on the NETTUNO model.

Nevertheless, one has to bear in mind that neither modern simulation techniques nor remote access to virtual laboratories do not eliminate necessity of conducting experiments in real laboratories with the use of real instruments. Practical experiments play an important role in the process of gaining knowledge in the field of modern, complex technologies that are conducted on the basis of 'trial and error' methods. This is of great importance whenever complex events cannot be described with the use of mathematical calculations.

\section{REFERENCES}

[1] L. Cristaldi, A. Ferrero, V. Piuri, "Programmable Instruments, Virtual Instruments and Distributed Measurement Systems, IEEE Instrumentation \& Measurement Magazine, vol.2, No.3, Sep., 1999, pp. 20-27.

[2] B. Galwas, S. Nowak, M. Pajer, P. Witonski, New Model of Electronic-Book for DistanceLearning Courses, Proceedings of the EDEN $10^{\text {th }}$ Anniversary Conference, Stockholm Sweden, 10-13 June 2001, pp. 465-468.

[3] B. Galwas, J. Barczyk, S. Nowak, E. Piwowarska, R. Rak, Web-based model of Engineering Studies Developed by Warsaw University of Technology, Proceedings of European Conference ,,The New Educational Benefits of ICT", 2-4 September, Rotterdam, 2002, pp. 95-98.

[4] B. Galwas, R. J. Rak, Virtual Laboratory - A Future Part Of The New Web-Based Model of Undergraduate Engineering Studies Developed By Warsaw University Of Technology, Joint IMEKO TC-1\& XXXIV MKM Conference 2002, 8 - 12 September 2002, pp. 58-63.

[5] H. Goldberg, What is virtual instrumentation, IEEE Instrumentation and Measurement
Magazine, vol.3, No. 4, December 2000, pp. 10-13.

[6] K. B. Lee, R. D. Schneeman, Internet-Based Distributed Measurement System and Control Application, IEEE Instrumentation \& Measurement Magazine, vol.2 No.3, June, 1999, pp. 23-27.

[7] F. Montesinos, J. Lacraz, C. Montforte, Case study on CEE Change: From face-to-face to open and distance learning CEE, Proceedings of the International Conference Paris-France, September 2000, CD-ROM.

[8] P. Pyszlak, R.J. Rak, A. Majkowski, The next approach to the design of a Web-based virtual laboratory, IEEE Instrumentation and Measurement Technology Conference, Vail, USA, May 2003, pp.1083-1086.

[9] R. J. Rak, Virtual Instrument - the Main Part of Internet Based Distributed System, International Conference on Advances in Infrastructure for Electronic Bussiness, Science, and Education on the Internet SSGRR'2000, L'Aquila, Italy, 2000, http://www.ssgrr.it/en/conferenza/index.htm.

[10] R. J. Rak, A. Michalski, Education in Instrumentation and Measurement: The Information and Communication Technology Trends, IEEE Instrumentation and Measurement Magazine, Vol.8, No.2, June 2005, pp. 61-69.

[11] R. J. Rak, M. Godziemba-Maliszewski, A. Majkowski, A proposal of Virtual Laboratory structure, Proceedings of IMTC 2006 Instrumentation and Measurement Technology Conference, Sorrento, Italy 24-27 April 2006.

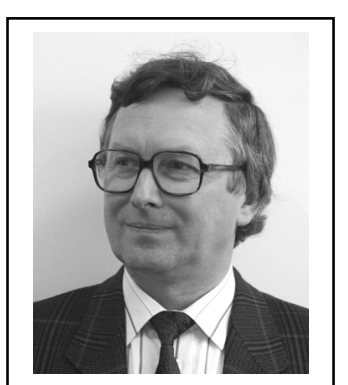

Remigiusz J. Rak, is a Professor of Electrical Engineering. He received the M.Sc., Ph.D. and D.Sc. degrees from Warsaw University of Technology in 1974, 1982 and 1998 respectively. In 2004 he got the title of professor. His current research interests include time-frequency analysis, signal compression, networked distributed measurement systems (Virtual Instruments and Internet based Virtual Laboratories) and distance learning models. Prof. Rak is a Head of the Department of Information and Measurement Systems at the Faculty of Electrical Engineering, Warsaw University of Technology. Currently he is also a Rector's Delegate to the New Forms and Innovations of Education. (rrak@okno.pw.edu.pl) 